\title{
Suppressing Deep Traps in PbS Colloidal Quantum Dots via Facile Iodide \\ Substitutional Doping for Solar Cells with Efficiency $>\mathbf{1 0} \%$
}

\author{
Alexandros Stavrinadis ${ }^{1}$, Santanu Pradhan ${ }^{1}$, Paris Papagiorgis ${ }^{2}$, Grigorios Itskos ${ }^{2}$, Gerasimos \\ Konstantatos ${ }^{1,3 *}$ \\ ${ }^{1}$ ICFO-Institut de Ciencies Fotoniques, The Barcelona Institute of Science and Technology, \\ 08860 Castelldefels (Barcelona), Spain \\ ${ }^{2}$ Department of Physics, Experimental Condensed Matter Physics Laboratory, University of \\ Cyprus, Nicosia, 1678, Cyprus \\ ${ }^{3}$ ICREA-Institució Catalana de Recerca i Estudis Avançats, Passeig Lluís Companys 23, \\ 08010 Barcelona, Spain \\ * gerasimos.konstantatos@icfo.es
}

\begin{abstract}
Surface passivation of PbS colloidal quantum dots (QDs) with iodide has been used in highly efficient solar cells. Iodide passivation is typically achieved by ligand exchange processes on QD films. Complementary to this approach, herein we present a non-intrusive solution-based strategy for doping QDs with iodide to further optimize solar cell performance. The doping step is applied in-situ at the end of the synthesis of the QDs. The optimum precursor $\mathrm{I} / \mathrm{Pb}$ ratio is found to be in the $1.5-3 \%$ range at which iodide substitutes S without excessively altering the dots' surface chemistry. This allows for band engineering and decreasing the density of deep trap states of the QDs which taken together lead to PbS QD solar cells with efficiency in excess of $10 \%$.
\end{abstract}




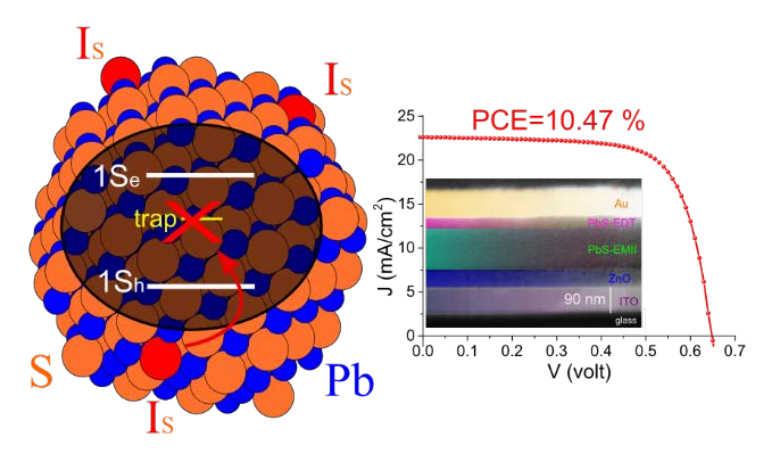

PbS QD based single junction solar cells have recently exceeded the $10 \%$ power conversion limit ${ }^{1}$. Much of this progress was facilitated by advancements made towards passivating the surface of the dots with ligands that cater for efficient inter-dot charge transfer while at the same time prevent the formation of surface-located electronic trap states ${ }^{2-4}$. Iodide and ethylenedithiol (EDT) are some of the most well studied ${ }^{5-8}$ and successful -in terms of end electronic functionality- ligands. These ligands combined are used for the fabrication of state of the art PbS CQD solar cells ${ }^{9}$. Therein, a bilayer of iodide-passivated dots and EDTpassivated dots form a respective type-II donor-acceptor interface with the term donor signifying: a) a negative shift of the valence and conduction band edges and b) an enhanced ntype doping character, with respect to the EDT-passivated dots. The shift of the band edges is related to changes of the ligand dependent electrostatic dipole moment on the dots' surface $^{9,10}$. The n-type doping effect of iodide dopants has been further assigned to two distinct mechanisms: i) $\mathrm{I}^{-}$substitutes divalent $\mathrm{S}^{-2}$ within the PbS structure, and ii) $\mathrm{I}^{-}$attached to the surface of the QDs prevents oxidation of the dots ${ }^{5}$. These two mechanisms are distinctive since, while the latter is a surface related one, the former is not necessarily surface located. 
Distinguishing the two mechanisms has not been a trivial task since iodide is usually introduced in abundance during the QD film formation ${ }^{5,6,11,12}$ aiming at passivating the exposed $\mathrm{Pb}$ surface sites. This approach further prevents combining the first mechanism (substituting $\mathrm{S}^{2-}$ ) with passivating the surface of the dots with another ligand, specifically with EDT. It is then possible to construct bilayers of iodide-passivated QDs and EDT-passivated QDs, where substitutional iodide dopants exist within each layer and counter-balance the negative impact that environmental oxidation ${ }^{5,13}$ and hydroxylation ${ }^{14}$ of the dots have on their ionic balance ${ }^{13}$ and thus doping character. Towards this direction, Lan et al. ${ }^{15}$ had recently reported such a doping method for photovoltaic applications were post-synthetically cleaned PbS QDs are subsequently doped over 24 hours in a solution containing a molecular $\mathrm{I}_{2}$ precursor under the inert $\mathrm{N}_{2}$ atmosphere of a glovebox. They further reported that at an optimized $\mathrm{I} / \mathrm{Pb}$ ratio of $5 \%$, the solar cell performance is statistically improved, and it drops above the aforementioned iodide concentration due to formation of traps as investigated by electronic device measurements ${ }^{15}$. We sought to develop a simpler and faster iodide doping method that would be readily integrated with all other steps taken in PbS QD photovoltaic technology, as well as to understand the physicochemical mechanisms at play that underpin the effects of this doping method on device performance.

The steps followed from QD synthesis to film formation for solar cells are tabulated in Figure 1a with step 2 being the one introduced in this work for the purpose of doping the QDs with iodide. After the synthetic reaction used for producing the QDs and before the cleaning step which typically involves the use of acetone, we introduce the iodide precursor which is 1ethyl-3-methylimidazolium iodide (EMII) dissolved at room temperature in acetone and further clean/process immediately afterwards. The EMII salt has been recently shown to be a more efficient iodide source for PbS QDs compared to the other commonly used TBAI ${ }^{14}$. In our method, to avoid excessive passivation of the dots surface with iodide and to sustaining 
colloidal stability of the QDs, a precursor ratio $\mathrm{I} / \mathrm{Pb} \leq 6 \%$ is used. When iodide doping density is kept at a moderate level of $\mathrm{I} / \mathrm{Pb}=1.5 \%$, the doped QDs exhibit enhanced photoluminescence compared to the un-doped ones as shown in Figure $1 \mathrm{~b}$ in agreement with prior reports ${ }^{15}$. From figure $1 \mathrm{~b}$ however, it is also evident that upon heavy doping $(\mathrm{I} / \mathrm{Pb}=6 \%)$ the PL intensity of the QDs is reduced. We further find that the aforementioned PL evolution with doping is accompanied by changes in the intensity of the Urbach Tail (UT) at the edge of the QDs' optical absorption spectra, as follows: for $\mathrm{I} / \mathrm{Pb}=1.5 \% \mathrm{UT}$ weakens compared to undoped QDs, while for heavy doping $(\mathrm{I} / \mathrm{Pb}=6 \%) \mathrm{UT}$ is re-strengthened, as shown in supplementary figure $\mathrm{S} 1 \mathrm{a}$. These facts (evolution of PL and UT with doping density) taken together indicate that at a moderate doping density, the density of empty trap states within the QDs' bandgap is reduced, but increases again for heavy iodide doping. We sought to confirm the implications of this phenomenon in PbS QD photovoltaic technology.

The QDs are used in solar cells employing $90 \mathrm{~nm}$ thick ITO, $60 \mathrm{~nm}$ thick $\mathrm{ZnO}$ and two $\mathrm{PbS}$ CQD film layers (of $200 \pm 10 \mathrm{~nm}$ total thickness) each one treated with EMII and EDT respectively followed by Au evaporation for the top electrodes. The cross section of the device is shown in Figure 1c. Typical current-voltage characteristics of these solar cells under 1-Sun illumination are shown in Figure 1d. The best photovoltaic performance of $10.5 \%$ is achieved at an optimum $\mathrm{I} / \mathrm{Pb}$ precursor ratio of $1.5 \%$, and at this doping density a robust statistical improvement of photovoltaic power conversion efficiency (PCE) is observed compared to solar cells made with un-doped dots as shown in Figures 1e,f. This improvement is facilitated by statistically higher open circuit voltage $\left(\mathrm{V}_{\mathrm{OC}}\right)$ and fill factor $(\mathrm{FF})$ values, but smaller $(<5 \%)$ short circuit ( $\mathrm{J}_{\mathrm{SC}}$ ) values, for cells with doped QDs compared to undoped QDs, as shown in supplementary Figure S2 which further includes average values and standard deviations for the aforementioned figures of merit. Statistically, the power conversion efficiency of the cells with 
optimized doped QDs is improved to $9.4 \pm 0.5 \%$ as compared to $8.9 \pm 0.6 \%$ for the undoped QDs.

Different precursor $\mathrm{I} / \mathrm{Pb}$ ratios were investigated in the $0-6 \%$ range and the best device results for each ratio are presented in Table 1 while the spectral photoresponse (external quantum efficiency i.e. EQE) of the devices is shown in supplementary Figure S3. The data in Table 1 illustrate that at heavy doping ( $/ \mathrm{Pb}=6 \%)$ the performance of the cells suffers from poor FF and Voc. Nevertheless, for all cells there is a good linear relationship between photocurrent and light intensity as shown in Supplementary Figure S4a. By employing transient photovoltage (TPV) and photocurrent (TPC) characterization, we find that the evolution of photovoltaic performance with increasing $\mathrm{I} / \mathrm{Pb}$ is associated with an initial decrease of trap state density and an increase of recombination lifetime as shown in Figures 2a,b. These trends however are reversed beyond the optimum I/Pb ratio as this reflects upon the deterioration of the solar cell performance for heavy iodide doping. We note that the same conclusions can further be confirmed by considering that at a given charge density value, charge recombination in the $\mathrm{I} / \mathrm{Pb}=0 \% \mathrm{QD}$ cell is faster (i.e. is described by smaller recombination rates) as compared to the $\mathrm{I} / \mathrm{Pb}>0 \%$ QD cells, and much faster compared to the optimized $\mathrm{I} / \mathrm{Pb}=1.5 \%$ QD cell. This is evident by Supplementary Figure S4b, which depicts the TPV-, and TPC- extracted recombination lifetime plotted against the photogenerated charge density. The photocurrent-time traces from the TPC characterization as shown in Supplementary Figures S4c,d also confirm that charge collection in the QD solar cells occurs faster $(<1 \mu \mathrm{s})$ compared to charge recombination which is typically characterized by $>1 \mu \mathrm{s}$ lifetimes as seen in Figure 2a.

Suppression of traps within the bandgap of the QDs via iodide doping should be an optically accessible effect and for this reason the PL properties of EMII-treated (i.e. ligand exchanged) QD films were investigated under low $(\approx 0.1$ excitons per QD) lighting conditions which are 
relevant to solar cell operation. The steady-state PL measurements shown in Figure 2c for different $\mathrm{I} / \mathrm{Pb}$ ratios, confirm that at optimized density $\mathrm{I} / \mathrm{Pb}=1.5 \%$ the $\mathrm{PL}$ strength is increased compared to the un-doped and heavily doped QDs. However, most importantly it is also observed (inset of Figure 2c) that an optimized iodide doping leads to elimination of the deep trap emission in the 1300-1600 nm range. This emission corresponds to optical transitions of energies $0.29-0.46 \mathrm{eV}$ below the band-edge emission of the QDs. Thus, this emission is facilitated by trap states located deep within the bandgap of the QDs. We hypothesize that for optimized iodide doping these states become partially filled by the electrons that become "free" when monovalent $\mathrm{I}^{-}$substitutes divalent $\mathrm{S}^{2-}$ in anion sites of the PbS structure. As a further consequence of this trap passivation mechanism, the PL-lifetime of the QDs is increased for $\mathrm{I} / \mathrm{Pb}=1.5 \%$ as evident from the transient $\mathrm{PL}$ measurements shown in Figure $2 \mathrm{~d}$. The deterioration of the QDs' PL characteristics for higher doping densities ( $\mathrm{I} / \mathrm{Pb}=6 \%$ ) should originate from the generation of new trap states upon heavy doping.

To further elucidate the physicochemical origins of the evolution of trap-density in QD films -and thus of their impact on the QDs' photovoltaic performance- with increasing I doping density, we employed X-ray and Ultraviolet photoelectron spectroscopies (XPS and UPS respectively) on EDT- and EMII-passivated QD films. The chemical species and their concentrations as identified by analysing the Pb4f, O1s, S2p, C1s and I3d XPS spectra are shown in Tables 2 and 3. Examples of deconvoluted XPS spectra are shown in Supplementary Figure S5 and analysed UPS spectra are shown in Supplementary Figure S6. For the EDTtreated QDs the following remarks can be made according to Table 2: i) XPS confirms the efficient incorporation of iodide dopants with our doping scheme and this is further seen in the I3d spectra shown in Figure 3a. ii) Iodide doping is accompanied by a decrease of the sulphur assigned to $\mathrm{PbS}$, this further indicates that iodide substitutes $\mathrm{S}$ in the $\mathrm{PbS}$ lattice; this mechanism should have an n-type doping effect on the QDs. iii) Iodide doping is accompanied 
by a decrease of EDT bound onto the QDs as measured from the S2p spectra. iv) At heavy iodide doping $(\mathrm{I} / \mathrm{Pb}=6 \%)$ undercharged $\mathrm{Pb}$ appears on the QDs and this can be a source of electronic trap states ${ }^{16}$. When calculating the ionic charge balance for the EDT films as shown in Table 2, we see that it progressively increases from 0.14 to 0.18 with increasing $\mathrm{I} / \mathrm{Pb}$. This should result to a progressive upward shift of the Fermi level $\left(E_{F}\right)$ with respect to the valence band edge $\left(\mathrm{E}_{\mathrm{V}}\right)$ of the QD film. Indeed, this is confirmed by the UPS measurements as shown in Figure 3b. The feature appearing on the Pb4f spectra and that is assigned to undercharged $\mathrm{Pb}$ is further shown in Figure 3c.

Figure 3b also shows the band levels for EMII-treated QD films with increasing the I/Pb precursor ratio of the initial doping step. Increasing $\mathrm{I} / \mathrm{Pb}$ does not monotonically cause an ntype doping effect (as determined by the relative position of $\mathrm{E}_{\mathrm{F}}$ compared to $\mathrm{E}_{\mathrm{C}}$ and $\mathrm{E}_{\mathrm{V}}$ ). Rather, $E_{F}$ does shift away from $E_{V}$ for the optimum doping $\mathrm{I} / \mathrm{Pb}=1.5 \%$, and then shifts backwards for heavy doping $(\mathrm{I} / \mathrm{Pb}=6 \%)$. This can be explained by considering the ionic charge balance as measured with XPS for these films and shown in Table 3. The stoichiometric data in Table 3 further suggest that the underlying reasons for the non-monotonic evolution of the charge balance calculation with increasing I/Pb for the EMII-treated QD films, are the following: i) The final iodide concentration of the films decreases when increasing the iodide concentration during the initial $\mathrm{I} / \mathrm{Pb}$ ratio. ii) While upon I-doping the concentration of $\mathrm{S}_{\mathrm{PbS}}$ is decreased, this effect does not progress further for heavy I-doping iii) After heavy doping, the final QD film are completely striped off the original oleic acid (as evident by the $\mathrm{O}_{\mathrm{C}=\mathrm{O}}$ concentration). This QD film is also significantly oxidized and hydroxylated as evident by both the $\mathrm{Pb}-\mathrm{O}$ and $\mathrm{Pb}-$ OH species' concentrations measured from the Pb4f and O1s XPS spectra. To account for the aforementioned effects, we posit that during the post-synthetic iodide doping, sulphur and oleic acid are removed and substituted -to some extent- by iodide. However, upon subsequent EMII/methanol treatment of the films, the following effects take place: methanol attacks and 
removes the originally attached (via substitution and/or surface attachment) iodide dopants and this renders the surface of the QDs prone to environmental oxidation and hydroxylation. These effects have a p-type doping effect which is confirmed by the evolution of $E_{F}$ as shown in Figure 3b, and can be explained by the charge balance calculations at the bottom of Table 3 . Our hypothesis further agrees with previous reports regarding the aggressive character of methanol in removing halides from the surface of $\mathrm{PbS} \mathrm{QDs}^{17}$.

Based on this analysis, it is evident that iodide doping of PbS QDs in solution improves solar cell performance as long as it mainly results to n-type substitutional doping and does not cause a significant alteration of the final QD films' surface chemistry. Such an alteration is the formation of undercharged lead in EDT-treated films which acts as a trap state and can be detrimental for solar cell performance ${ }^{16}$. For EMII-treated films, the undesired side effect of the initial iodide doping is the oxidation and hydroxylation of the final QD films, with both mechanisms having p-type doping effect and introducing electronic trap states ${ }^{14,18}$. The doping effect and chemical side effects of the iodide doping scheme presented here when followed by EDT and EMII treatment of the QD films, are tabulated in Figure 3d. When an optimum I/Pb ratio of $1.5 \%$ is used and the detrimental surface effects are thus being avoided, the solar cells are benefited by iodide substitutional doping. Furthermore, at the optimum doping concentration, the band edge offset between the EMII- and EDT-treated QD films is at its maximum as can be seen in Figure 3b. This may further account for the improved performance and $\mathrm{V}_{\mathrm{OC}}$ of the cells made with optimized doped QDs.

In conclusion, subtle changes in the iodide doping density of PbS QDs in solution may alter significantly the chemistry of the final QD films. This in turn affects the electronic structure, density of trap states, photoluminescence intensity and dynamics, carrier recombination lifetime and photovoltaic performance of the QD films. The efficiency of solar cells is improved for an optimized iodide doping density for which a clear substitutional doping 
mechanism is resolved. When exceeding the optimum doping density, the photovoltaic performance of the QD films drops due to undesired chemical species formed on the surface of the QDs films.

\section{Supporting Information (SI)}

Supporting Information Available: Chemicals used, Experimental Methods for i) the synthesis of PbS QDs, ii) the solution based iodide doping, iii) synthesis of zinc oxide nanoparticles $^{19}$, iv) fabrication of solar cells, v) characterization of solar cells, vi) optical

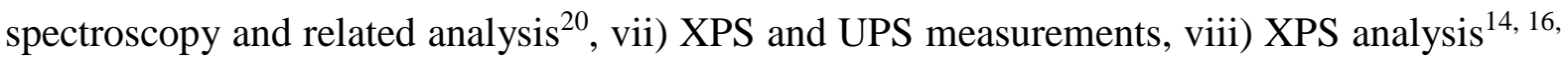
${ }^{21-22}$. SI also includes absorption spectra of QD films, histogram plots for figures of merit of solar cells, plots of external quantum efficiency of solar cells and related discussion ${ }^{23}$, XPS and UPS spectra of QD films.

\section{Acknowledgments}

We acknowledge financial support from the European Research Council (ERC) under the European Union's Horizon 2020 research and innovation programme (grant agreement No 725165), the Spanish Ministry of Economy and Competitiveness (MINECO) and the "Fondo Europeo de Desarrollo Regional” (FEDER) through grant MAT2014-56210-R. This work was also supported by AGAUR under the SGR grant (2014SGR1548) and by the EC under the Graphene Flagship (contract no. CNECT-ICT-604391). We also acknowledge financial support from Fundacio Privada Cellex and from the Spanish Ministry of Economy and Competitiveness, through the "Severo Ochoa" Programme for Centres of Excellence in R\&D (SEV-2015-0522). 


\section{References}

(1) Lan, X.; Voznyy, O.; García de Arquer, F. P.; Liu, M.; Xu, J.; Proppe, A. H.; Walters, G.; Fan, F.; Tan, H.; Liu, M.; et al. 10.6\% Certified Colloidal Quantum Dot Solar Cells via Solvent-Polarity-Engineered Halide Passivation. Nano Lett. 2016, 16, 4630-4634.

(2) Crisp, R. W.; Kroupa, D. M.; Marshall, A. R.; Miller, E. M.; Zhang, J.; Beard, M. C.; Luther, J. M. Metal Halide Solid-State Surface Treatment for High Efficiency PbS and PbSe QD Solar Cells. Sci. Rep. 2015, 5, 9945.

(3) Ip, A. H.; Thon, S. M.; Hoogland, S.; Voznyy, O.; Zhitomirsky, D.; Debnath, R.; Levina, L.; Rollny, L. R.; Carey, G. H.; Fischer, A.; et al. Hybrid Passivated Colloidal Quantum Dot Solids. Nature Nanotechnology 2012, 7, 577-582.

(4) Zhang, N.; Neo, D. C. J.; Tazawa, Y.; Li, X.; Assender, H. E.; Compton, R. G.; Watt, A. A. R. Narrow Band Gap Lead Sulfide Hole Transport Layers for Quantum Dot Photovoltaics. ACS Appl. Mater. Interfaces 2016, 8, 21417-21422.

(5) Zhitomirsky, D.; Furukawa, M.; Tang, J.; Stadler, P.; Hoogland, S.; Voznyy, O.; Liu, H.; Sargent, E. H. N-Type Colloidal-Quantum-Dot Solids for Photovoltaics. Adv. Mater. 2012, 24, 6181-6185.

(6) Tang, J.; Kemp, K. W.; Hoogland, S.; Jeong, K. S.; Liu, H.; Levina, L.; Furukawa, M.; Wang, X.; Debnath, R.; Cha, D.; et al. Colloidal-Quantum-Dot Photovoltaics Using Atomic-Ligand Passivation. Nat. Mater. 2011, 10, 765-771.

(7) Gao, J.; Jeong, S.; Lin, F.; Erslev, P. T.; Semonin, O. E.; Luther, J. M.; Beard, M. C. Improvement in Carrier Transport Properties by Mild Thermal Annealing of $\mathrm{PbS}$ Quantum Dot Solar Cells. Appl. Phys. Lett. 2013, 102, 043506.

(8) Barkhouse, D. A. R.; Pattantyus-Abraham, A. G.; Levina, L.; Sargent, E. H. Thiols Passivate Recombination Centers in Colloidal Quantum Dots Leading to Enhanced Photovoltaic Device Efficiency. ACS Nano 2008, 2, 2356-2362.

(9) Chuang, C.-H. M.; Brown, P. R.; Bulović, V.; Bawendi, M. G. Improved Performance and Stability in Quantum Dot Solar Cells through Band Alignment Engineering. Nat. Mater. 2014, 13, 796-801.

(10) Brown, P. R.; Kim, D.; Lunt, R. R.; Zhao, N.; Bawendi, M. G.; Grossman, J. C.; Bulović, V. Energy Level Modification in Lead Sulfide Quantum Dot Thin Films through Ligand Exchange. ACS Nano 2014, 8, 5863-5872.

(11) Ning, Z.; Ren, Y.; Hoogland, S.; Voznyy, O.; Levina, L.; Stadler, P.; Lan, X.; Zhitomirsky, D.; Sargent, E. H. All-Inorganic Colloidal Quantum Dot Photovoltaics Employing Solution-Phase Halide Passivation. Adv. Mater. 2012, 24, 6295-6299. 
(12) Milliron, D. J. Quantum Dot Solar Cells: The Surface Plays a Core Role. Nat Mater. 2014, 13, 772-773.

(13) Voznyy, O.; Zhitomirsky, D.; Stadler, P.; Ning, Z.; Hoogland, S.; Sargent, E. H. A Charge-Orbital Balance Picture of Doping in Colloidal Quantum Dot Solids. ACS Nano 2012, 6, 8448-8455.

(14) Cao, Y.; Stavrinadis, A.; Lasanta, T.; So, D.; Konstantatos, G. The Role of Surface Passivation for Efficient and Photostable PbS Quantum Dot Solar Cells. Nat. Energy 2016, 1, 16035.

(15) Lan, X.; Voznyy, O.; Kiani, A.; García de Arquer, F. P.; Abbas, A. S.; Kim, G.-H.; Liu, M.; Yang, Z.; Walters, G.; Xu, J.; et al. Passivation Using Molecular Halides Increases Quantum Dot Solar Cell Performance. Adv. Mater. 2016, 28, 299-304.

(16) Hwang, G. W.; Kim, D.; Cordero, J. M.; Wilson, M. W. B.; Chuang, C.-H. M.; Grossman, J. C.; Bawendi, M. G. Identifying and Eliminating Emissive Sub-Bandgap States in Thin Films of PbS Nanocrystals. Adv. Mater. 2015, 27, 4481-4486.

(17) Kirmani, A. R.; Carey, G. H.; Abdelsamie, M.; Yan, B.; Cha, D.; Rollny, L. R.; Cui, X.; Sargent, E. H.; Amassian, A. Effect of Solvent Environment on Colloidal-Quantum-Dot Solar-Cell Manufacturability and Performance. Adv. Mater. 2014, 26, 4717-4723.

(18) Konstantatos, G.; Levina, L.; Fischer, A.; Sargent, E. H. Engineering the Temporal Response of Photoconductive Photodetectors via Selective Introduction of Surface Trap States. Nano Lett. 2008, 8, 1446-1450.

(19) Beek, W. J. E.; Wienk, M. M.; Kemerink, M.; Yang, X.; Janssen, R. A. J. Hybrid Zinc Oxide Conjugated Polymer Bulk Heterojunction Solar Cells. J. Phys. Chem. B 2005, 109, 9505-9516.

(20) Lakowicz, J. R. in Principles of Fluorescence Spectroscopy $3^{\text {rd }}$ edn, Springer, Chapter 4, New York 2006

(21) Laajalehto, K.; Kartio, I.; Nowak, P. XPS Study of Clean Metal Sulfide Surfaces. Appl. Surf. Sci. 1994, 81, 11-15.

(22) Luther, J. M.; Law, M.; Song, Q.; Perkins, C. L.; Beard, M. C.; Nozik, A. J. Structural, Optical, and Electrical Properties of Self-Assembled Films of PbSe Nanocrystals Treated with 1,2-Ethanedithiol. ACS Nano 2008, 2, 271-280.

(23) Willis, S. M.; Cheng, C.; Assender, H. E; Watt, A.A.R. The Transitional Heterojunction Behavior of PbS/ZnO Colloidal Quantum Dot Solar Cells. Nano Lett. 2012, 12, 15221526. 
Table 1. Best results for solar cells made with QDs of different I/Pb doping ratios.

\begin{tabular}{|c|c|c|c|c|c|c|}
\hline Precursor I/Pb (\%) & $\mathrm{V}_{\mathrm{OC}}(\mathrm{V})$ & $\mathrm{J}_{\mathrm{SC}}\left(\mathrm{mA} / \mathrm{cm}^{2}\right)$ & $\mathrm{FF}$ & $\mathrm{PCE}(\%)$ & $\mathrm{R}_{\mathrm{s}}(\mathrm{Ohm})$ & $\mathrm{R}_{\mathrm{sh}}(\mathrm{Ohm})$ \\
\hline 0 & 0.64 & 22 & 71 & 9.96 & 19.7 & 62900 \\
\hline 0.75 & 0.62 & 21.8 & 71 & 9.62 & 23.5 & 18400 \\
\hline 1.5 & 0.65 & 22.6 & 71 & 10.47 & 25.9 & 50900 \\
\hline 3 & 0.66 & 21.3 & 72 & 10.05 & 44.8 & 31600 \\
\hline 6 & 0.63 & 21.2 & 63 & 8.82 & 48.3 & 14600 \\
\hline
\end{tabular}


Table 2. XPS results for EDT treated samples. For $\mathrm{Pb} 4 \mathrm{f}$ and S2p peak energies refer to $\mathrm{Pb} 4 \mathrm{f} 5 / 2$ and S2p3/2 respectively.

\begin{tabular}{|c|c|c|c|c|}
\hline ElementXPS peak & Speciesbinding energy & $\mathrm{I} / \mathrm{Pb}=0 \%$ & $\mathrm{I} / \mathrm{Pb}=1.5 \%$ & $\mathrm{I} / \mathrm{Pb}=6 \%$ \\
\hline \multirow[t]{4}{*}{$\mathrm{Pb}_{4 \mathrm{f}}$} & Total & 1 & 1 & 1 \\
\hline & $\mathrm{PbS}_{137.5 \mathrm{eV}}$ & 0.96 & 0.96 & 0.91 \\
\hline & $\mathrm{Pb} / \mathrm{PbS}$ oxidized $138.4 \mathrm{eV}$ & 0.04 & 0.04 & 0.03 \\
\hline & $\mathrm{Pb}$ undercharged $136.4 \mathrm{eV}$ & - & - & 0.06 \\
\hline \multirow[t]{4}{*}{$S_{2 p}$} & Total & 1.24 & 1.21 & 1.14 \\
\hline & $\mathrm{PbS}_{160.6 \mathrm{eV}}$ & 0.62 & 0.6 & 0.58 \\
\hline & EDT bound $_{161.5 \mathrm{eV}}$ & 0.57 & 0.53 & 0.53 \\
\hline & EDT unbound $163.5 \mathrm{eV}$ & 0.06 & 0.08 & 0.02 \\
\hline $\mathrm{I}_{3 \mathrm{~d}}$ & $\mathrm{PbI}_{618.7 \mathrm{eV}}$ & 0 & 0.01 & 0.05 \\
\hline \multirow[t]{2}{*}{$\mathrm{C}_{1 \mathrm{~s}}$} & Total & 1.03 & 1.10 & 1.31 \\
\hline & $\mathrm{C}=\mathrm{O}_{288.9 \mathrm{eV}}$ & 0.04 & 0.05 & 0.05 \\
\hline \multirow[t]{5}{*}{$\mathrm{O}_{1 \mathrm{~s}}$} & Total & 0.12 & 0.18 & 0.11 \\
\hline & $\mathrm{Pb}-\mathrm{O}_{529.6 \mathrm{eV}}$ & 0.01 & 0.01 & 0 \\
\hline & $\mathrm{Pb}-\mathrm{OH}_{531.2 \mathrm{eV}}$ & 0.03 & 0.08 & 0.02 \\
\hline & $\mathrm{COO}, \mathrm{CO} 2532.3 \mathrm{eV}$ & 0.04 & 0.05 & 0.05 \\
\hline & $\mathrm{OH}_{533.7 \mathrm{eV}}$ & 0.04 & 0.04 & 0.05 \\
\hline $\begin{array}{l}\text { Charge balance } \\
\text { according to XPS }\end{array}$ & 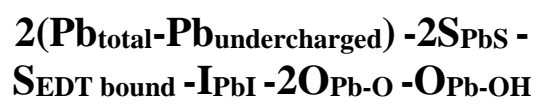 & 0.14 & 0.16 & 0.18 \\
\hline
\end{tabular}


Table 3. XPS results for EMII treated samples

\begin{tabular}{|c|c|c|c|c|}
\hline ElementXPS peak & Speciesbinding energy & $\mathrm{I} / \mathrm{Pb}=0 \%$ & $\mathrm{I} / \mathrm{Pb}=1.5 \%$ & $\mathrm{I} / \mathrm{Pb}=6 \%$ \\
\hline \multirow[t]{3}{*}{$\mathrm{Pb}_{4 \mathrm{f}}$} & Total & 1 & 1 & 1 \\
\hline & $\mathrm{PbS}_{137.9 \mathrm{eV}}$ & 0.95 & 0.95 & 0.92 \\
\hline & $\mathrm{Pb} / \mathrm{PbS}$ oxidized $_{138.9 \mathrm{eV}}$ & 0.05 & 0.05 & 0.08 \\
\hline$S_{2 p}$ & $\mathrm{PbS}_{161 \mathrm{eV}}$ & 0.61 & 0.59 & 0.59 \\
\hline $\mathrm{I}_{3 \mathrm{~d}}$ & $\mathrm{PbI}_{619.2 \mathrm{eV}}$ & 0.64 & 0.63 & 0.61 \\
\hline \multirow[t]{2}{*}{$\mathrm{C}_{1 \mathrm{~s}}$} & Total & 0.67 & 0.70 & 0.89 \\
\hline & $\mathrm{C}=\mathrm{O}_{288.9 \mathrm{eV}}$ & 0.04 & 0.04 & $<0.01$ \\
\hline \multirow[t]{5}{*}{$\mathrm{O}_{1 \mathrm{~s}}$} & Total & 0.14 & 0.13 & 0.18 \\
\hline & $\mathrm{Pb}-\mathrm{O}_{529.6 \mathrm{eV}}$ & 0.01 & 0.01 & 0.04 \\
\hline & $\mathrm{Pb}-\mathrm{OH}_{531.2 \mathrm{eV}}$ & 0.03 & 0.02 & 0.07 \\
\hline & $\mathrm{COO}, \mathrm{CO} 2_{532.3 \mathrm{eV}}$ & 0.05 & 0.05 & 0.04 \\
\hline & $\mathrm{OH}_{533.7 \mathrm{eV}}$ & 0.05 & 0.05 & 0.03 \\
\hline $\begin{array}{l}\text { Charge balance } \\
\text { according to XPS }\end{array}$ & $\begin{array}{c}2 P_{\text {total }}-2 S_{P b S}-I_{P b I}- \\
2 O P b-O-O P b-O H\end{array}$ & 0.09 & 0.15 & 0.06 \\
\hline
\end{tabular}




\section{Figures}

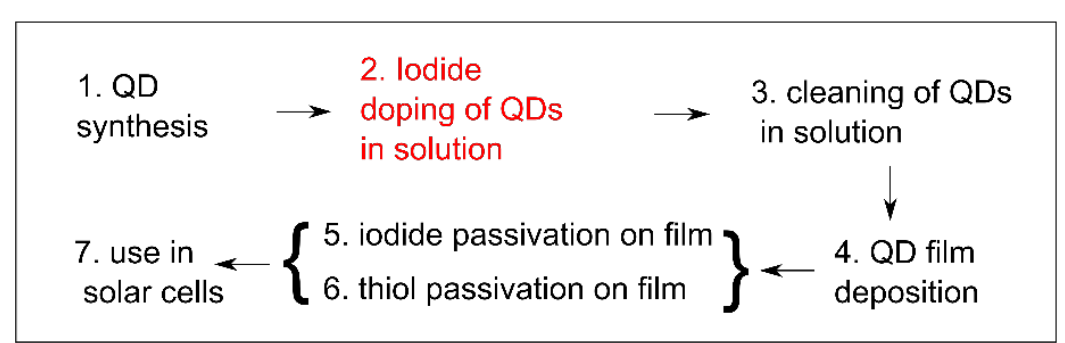

(a)

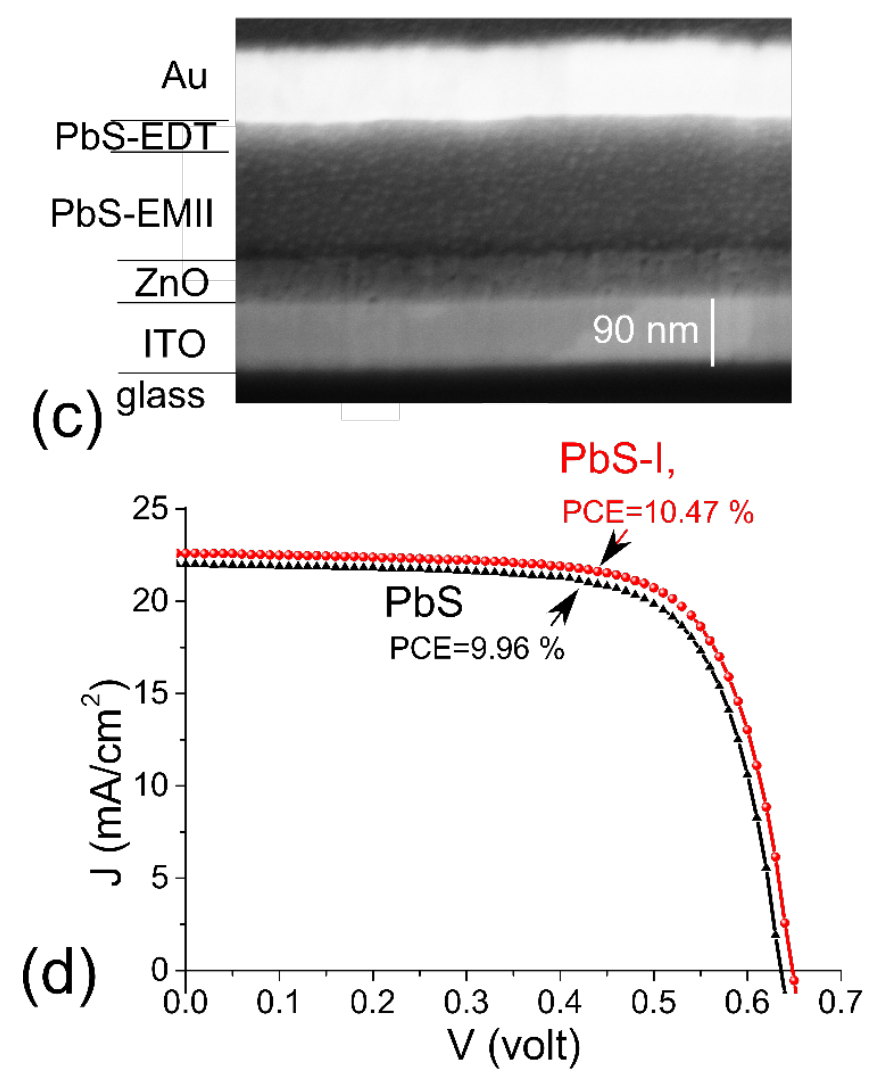

(b)
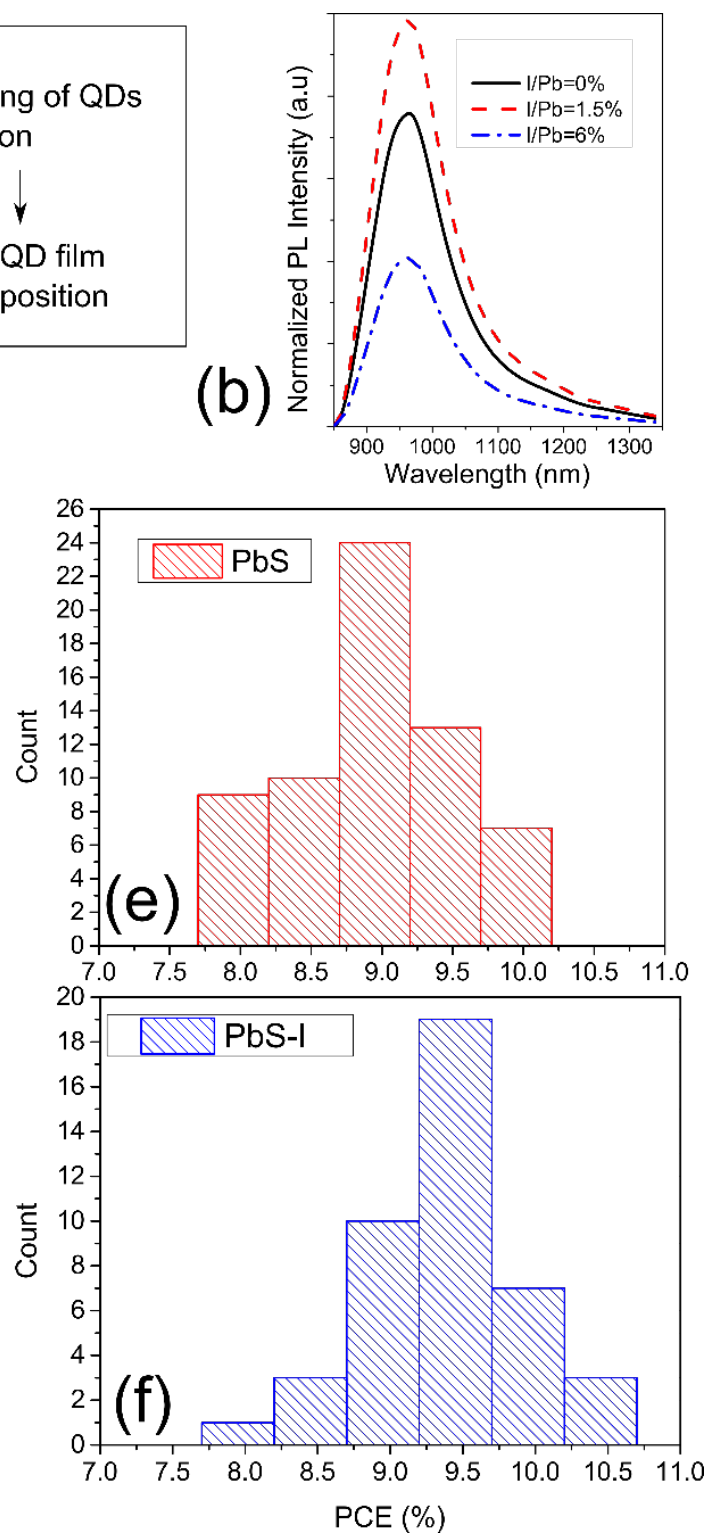

Figure 1 (a) CQD processing towards use in solar cells, (b) Normalized (according to optical absorption) PL profile upon increasing iodide doping concentration in solution (no additional ligand exchange), (c) micrograph of the cross section of a QD solar cell with layers as indicated, (d) current-voltage trace under 1-sun illumination for best solar cells made without (black line) and with (red line) halide doping and indicated power conversion efficiency (PCE) values, (e,f) histograms for PCE of several solar cells made without (e) and with (f) optimized halide doping of QDs in solution. 

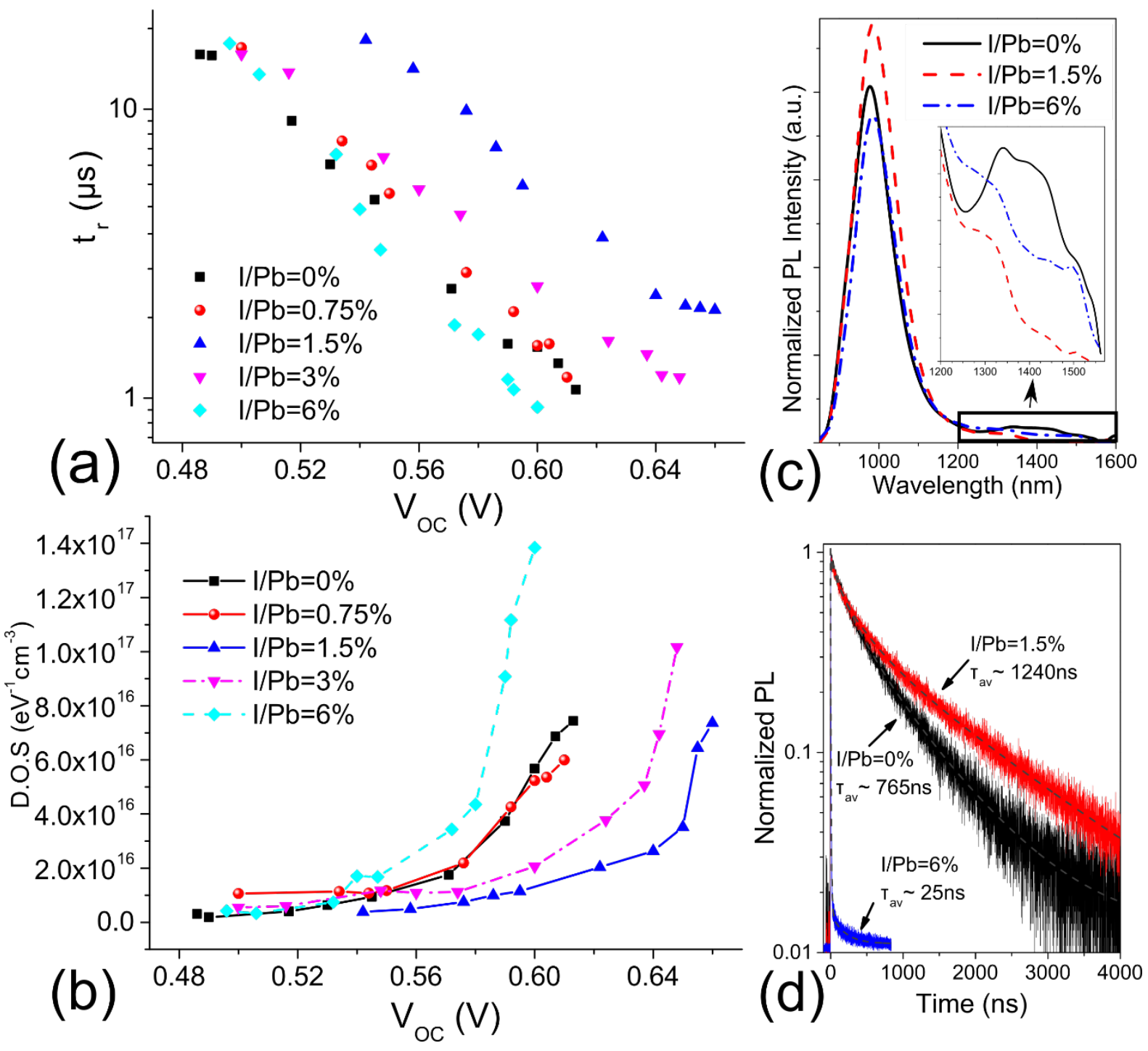

Figure 2 (a) Carrier recombination lifetime and (b) density of trap states, in solar cells for different $\mathrm{I} / \mathrm{Pb}$ doping ratios. (c) Steady-state PL (normalized to absorption) measurements of EMII-treated films of QDs of different initial I/Pb doping ratio. The region in the 1200-1600 nm range, is further shown in the inset. (d) Normalized PL lifetime traces for the EMII-treated QD films and indicated average lifetimes as calculated by a triple-exponential fitting (dashed lines) performed for each trace. [Measurements shown in (c) and (d) were performed using laser excitation at $785 \mathrm{~nm}$ and an excitation intensity corresponding to $\approx 0.1$ excitons per QD.] 


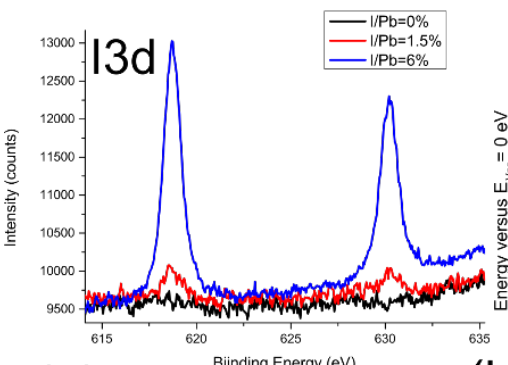

(a)

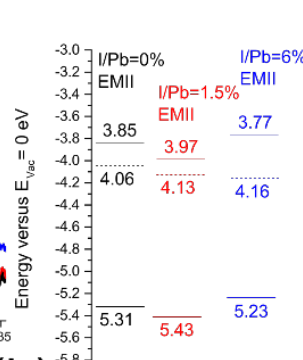

(b)

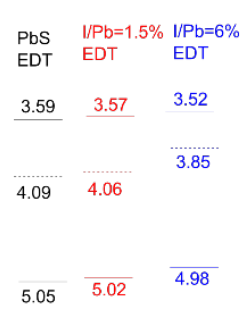

(c)
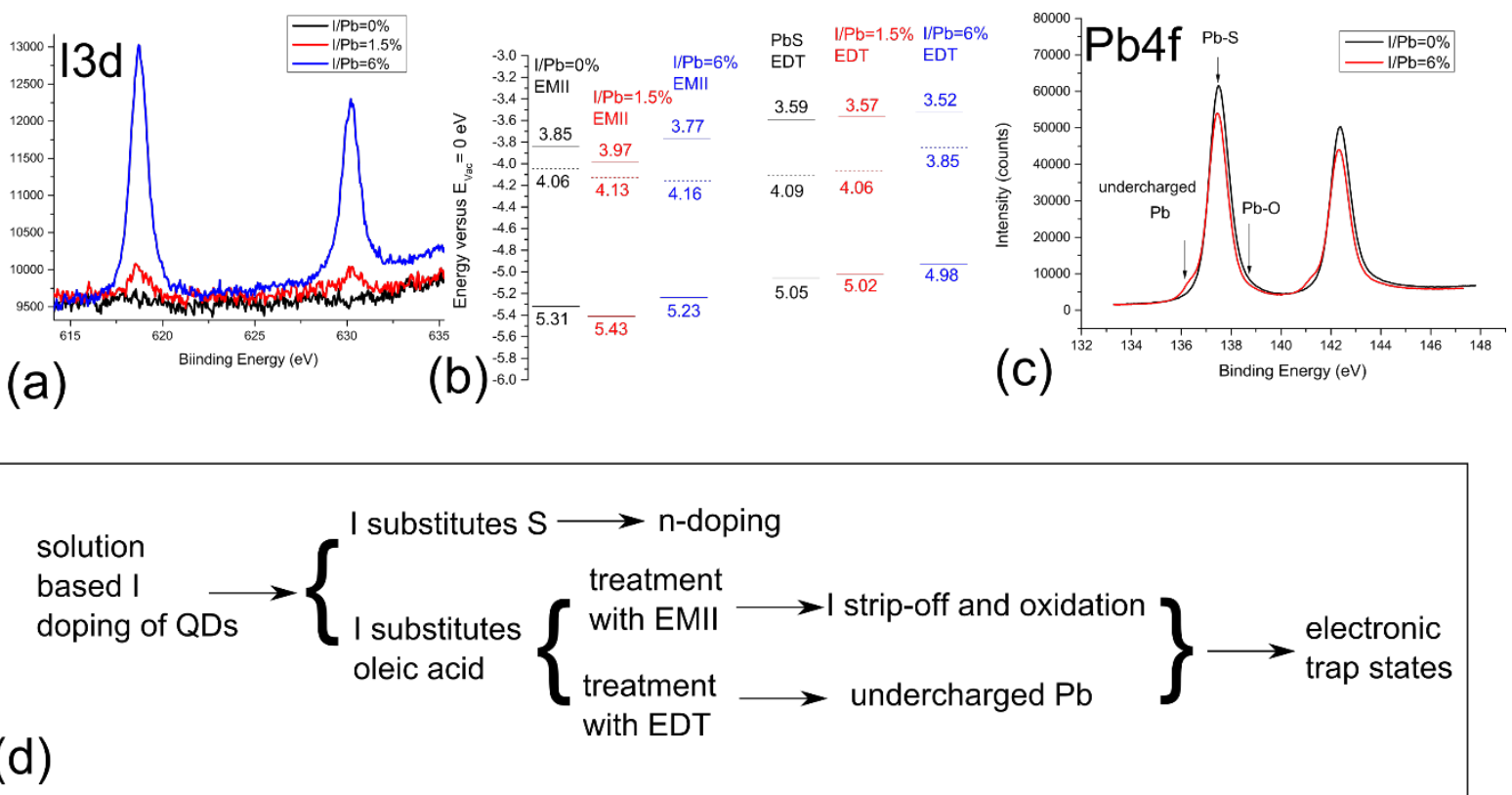

Figure 3. (a) I3d XPS spectra of EDT-treated QD films with varying I/Pb precursor ratio. (b) Energy positions of valence band edge $\mathrm{E}_{\mathrm{V}}$ (lower solid lines), conduction band edge $\mathrm{E}_{\mathrm{C}}$ (upper solid lines) and Fermi level (dotted lines) according to UPS measurements and bandgap of QDs, for EMII- and EDT-treated QD films. (c) Pb4f XPS spectra of EDT-treated films of undoped and heavily iodide-doped QDs. (d) Schematic showing the electronic doping effect and chemical and electronic side effects of the iodide doping scheme presented here. 